\title{
The liver
}

$\mathrm{T}$ his conference was attended by over 600 delegates. The theme that pervaded most sessions was molecular biology; hence the meeting clearly had a basic science rather than a clinical bias. Taking into account the author's own bias in hepatology, this is a brief report on new and/or interesting topics discussed at this meeting.

A full day satellite symposium on primary biliary cirrhosis (PBC) was held which prompted the comment that soon there would be more papers on $\mathrm{PBC}$ than patients! The hot topic for debate was the origin and specificity of the antimitochondrial antibody (AMA). Drs Fussey and M Bassendine have identified the antigenic components responsible for this marker antibody as the acetyltransferase portion on the inner membrane of mitochondria. PBC sera react with the lipoyl domain of this antigen. A similar lipoyl domain may also be found in bacterial 2-oxoacid dehydrogenase complexes, suggesting that reactivity of $\mathrm{PBC}$ sera with rough mutants of Escherichia coli is likely due to cross-reactivity and does not indicate an etiological role for this bacteria in PBC. Dr Gerschwin described experiments in which animals could be immunized to produce antibodies to mitochondrial fractions, but no PBC-like lesions were identified in the liver. Dr Frank Chisari has shown that lymphocyte extracts from the liver or lymph nodes but not peripheral lymphocytes of PBC patients, when given with interleukin-2 and mitochondrial antigens to mice, lead to the development of a liver lesion similar to PBC; however, the histological pattern is not dissimilar to graft versus host reaction. There is still no evidence that AMA plays any role in the etiology of $\mathrm{PBC}$, but the identification of factors involved in its production sheds further light on the pathophysiology of this disease. Dr David Triger provided some interesting data on AMA which demonstrated that AMA was equally prevalent in blood relatives of index $\mathrm{PBC}$ cases $(7.6 \%$ positive) and spouses ( $7.2 \%$ positive). This observation lends support to his other data suggesting the role of the environment in the prevalence of PBC.

Dr J Boyer, this year's IASL president, gave an excellent review of the natural history of $\mathrm{PBC}$. The data were collected from the very large PBC patient population ( 300 plus) referred to the Yale group over the past 30 to 40 years. His data suggest that the mean survival of symptomatic PBC patients from time of initial presentation is 10.1 years, whereas it is 17.8 years for asymptomatic cases. Sixty-three per cent of these 36 asymptomatic patients became symptomatic over 20 years; 15 died, $73 \%$ from liver disease. It seems that survival beyond 12 years is the cut-off point at which the outcome of an asymptomatic case is worse than the age and sex-matched population.

The only other new data came from Dr Roger Williams of the United Kingdom, who claimed that bone density follow. ing liver transplantation for PBC decreased postoperatively but began to increase six months after transplantation.

The postgraduate course consisted of a series of fairly simplified illustrations of the role of molecular biological techniques as they could be applied to the study of liver diseases. The talk by Dr Kim Summers on molecular genetics did much to clarify the subsequent papers. There was an excellent paper from Newcastle, United Kingdom showing differing $\mathrm{ADH} 3$ allele frequencies in patients with alcohol-related liver disease compared with controls. Unfortunately, the control group did not include alcoholics without liver disease - a difficult population to identify accurately. Another paper from the same group beautifully demonstrated that the $\mathrm{Pi}$, $\mathrm{PiS}$ and PiM variants of alpha-1 antitrypsin deficiency could be differentiated by rates of unfolding to the native forms and refolding on polyacrylamide gel electrophoresis (PAGE) using different concentrations of urea when electrophoresis in PAGE was performed perpendicular to the gradient. They suggest that these differences may account for the variable expression of deficiency seen on liver biopsy. A further paper using molecular genetics, from Omata's group in Japan, indicated that ras gene mutations seen frequently in colonic and pancreatic cancer could also be found in cholangiocarcinoma but not in hepatoma, suggesting that a toxin which could cause such a point mutation may be important in the pathogenesis of cholangiocarcinoma in Japan. 
Dr Rudi Schmid, in his summary on basic scientific perspectives for the future of hepatology, suggested that recognition of polygenicity for diseases and gene transfer techniques were likely to gain importance. Dr Sheila Sherlock, in summing up her ideas on the future of clinical hepatology, stressed the importance of advocating universal hepatitis B vaccination, better vaccines and development of a hepatitis $\mathrm{C}$ vaccine with pre-S1 components for $\mathrm{B}$, better antiviral agents, earlier detection and treatment of hepatocellular cancer and the development of noninvasive techniques to identify liver disease and fibrosis. Two tests, one for serum hyaluronate (from Dr Rudi Preisig's group in Berne, Switzerland) and one for protein F (from Dr Oliver James's group in Newcastle, United Kingdom), claimed to do just that.

A major portion of the meeting was devoted to hepatitis C. Dr Harvey Alter pointed out that the hepatitis C virus antibody test currently available would soon be replaced by a second generation test that detected both the $\mathrm{C} 100$ and $\mathrm{C} 33$ fractions, thereby increasing the sensitivity of the test. He gave more data on the correlation of the polymerase chain reaction (PCR) test for viral RNA with hepatitis $\mathrm{C}$ virus antibodies - the two are nearly always positive together, ie, the virus remains present for several years after the initial infection but may eventually disappear from the serum (the PCR test which detects different epitopes from the serum test on liver tissue is not well developed at present). It is possible that some, but likely few truly hepatitis $\mathrm{C}$ virus antibody positive sera, are not infectious. Blood positive for hepatitis $\mathrm{C}$ virus antibody by enzyme-linked immunosorbent assay (ELISA) and recombinant immunoblot assay (RIBA) transfers infection 72 to $80 \%$ of the time, if transfused. However, many healthy blood donors ELISA positive for hepatitis C virus antibodies are RIBA negative; there is a high false-positive rate in blood donors. The same high false-positive rate is not found in subjects with liver disease.

There were several papers on the value of interferon treatment in chronic hepatitis $\mathrm{C}$ - the bottom line is that $3 \times 10^{6}$ units given daily or more frequently is better than $1 \times 10^{6}$ units, and treatment should probably be extended to 12 months to obtain sustained remission (ie, normalization of serum alanine aminotransferase) in 40 to $60 \%$. Clear predictors of a good response have not been identified, although a poster from Dr M Rizzetto's group in Turin, Italy suggested that female sex, baseline low autoantibodies and absence of cirrhosis were associated with a better long term outcome. However, the presence or absence of hepatitis $\mathrm{C}$ virus antibody is no predictor, and few studies on the PCR RNA test are available. A statement at question time claimed that the disappearance of a positive PCR test was associated with a fall in liver enzymes and clinical relapse associated with the reappearance of a positive PCR test. Dr Hoofnagle gave two warnings with regard to interferon side effects in the treatment of chronic non- $A$,non- $B$ hepatitis. The first was that clinically significant autoimmune disease, particularly thyroid, had now been reported several times, and that jaundice occurring shortly after the introduction of interferon therapy may indicate that the patient actually has autoimmune hepatitis and interferon must be stopped immediately. Unlike patients with decompensated hepatitis $B$, hepatitis $C$ patients with equally severe disease may respond positively to interferon therapy. There was one poster from Japan reporting the use of long acting interferon (given monthly). This mini-pellet preparation sounds promising! The general consensus on hepatitis C antibody testing in chronic liver disease was that false positives were rare if careful testing was performed. The very expensive RIBA test can be used, not so much as a confirmatory test, but to rule out false positives.

Hepatitis B took second place to hepatitis C at this meeting. The hottest topic for discussion in the field of hepatitis B was viral mutants. Several have been described: hepatitis B surface antigen positive with no anti-hepatitis $B$ coat antigen or anti-E; anti-E positive hepatitis B virus DNA positive (ie, the precore variant); and more recently, hepatitis B surface antigen positive, E positive but no anti-hepatitis B coat antigen in a human immunodeficiency virus positive patient. The role of interferon therapy in the precore mutant disease is still undecided; at present high relapse rates are described. There was much debate about whether chronic hepatitis B patients should be primed with a pulse of high dose prednisone prior to interferon therapy. The general consensus was that this could be dangerous and precipitate a severe flare-up, particularly in the cirrhotic patient, but that it may be the therapy with the greatest chance of being successful in the patient infected very early in life. Dr Rizzetto pointed out that interferon therapy in hepatitis B patients co-infected with delta needs to be prolonged, perhaps for several years, and is only effective if hepatitis B surface antigen is eradicated. He also stated that hepatitis B patients co-infected with delta had a good two year post transplant survival if hepatitis B surface antigen could be eradicated with high dose anti-hepatitis B surface antigen despite remaining delta positive, ie, delta is noncytopathic in the absence of hepatitis B surface antigen. The current opinion on interferon treatment for chronic hepatitis B subjects co-infected with human immunodeficiency virus is that if immunosuppression is not fully developed, ie, normal T4 cell numbers, interferon therapy may be worthwhile.

The lack of new reports on alcoholic liver disease, portal hypertension, hepatic encephalopathy and ascites (the usual mainstays of liver meetings) was obvious. Instead, there were excellent preparations on the genetic basis of hepatic drug metabolism given by Dr U Meyer. He described the common genetic problems of drug metabolism, eg, slow acetylation and altered c-oxidation, which may affect significant proportions of the population and be responsible for adverse drug reactions (isoniazid), lack of efficacy (inability to convert codeine to morphine), and certain disease states. The significance of the cytochrome P450 antibody found in the serum of children with autoimmune hepatitis remains unknown. Dr Irwin Arias, during his talk on multidrug resistance and primary hepatocellular carcinoma, showed evidence that indicated that the calcium channel blockers may reverse multiple drug resistance - and that trials of verapamil given with multiple chemotherapy regimens are now in progress. The only new 
item in the management of gallstones was the observation that lovastatin decreases the cholesterol saturation of bile, and given in combination with ursodeoxycholic acid the effect is synergistic. Dr M Carey of Boston, who delivered a gallstone talk with his usual eloquence, discussed the role of lecithin in hepatobiliary physiology. It seems that once lecithin enters the gallbladder it becomes converted to prostanoids in the mucosa (the latter are thought responsible for the secretion of mucin glycoproteins, potential nucleating agents), which appear to affect gallbladder motility.

The era of the endothelial cell is well established. The role of the lto cell (lipocyte) was addressed by Dr M Bissell of the United States. He described a close interaction between the
Kupffer cell, lipocytes and activation of the latter occurring via platelet activating factor. Whether lymphokines activate lipocytes directly or via Kupffer cells is unknown.

There were many other fine speakers whose work is not mentioned in this review because of lack of space and author bias. Clearly molecular biology is going to play an increasingly important role in the understanding of disease and it behooves all to become more familiar with some of the basic principles of this exciting science.

Jenny Heathcote, MB, BS, FRCPC Toronto Western Hospital Toronto, Ontario 


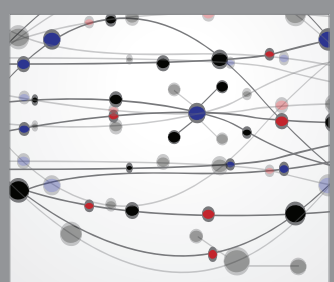

The Scientific World Journal
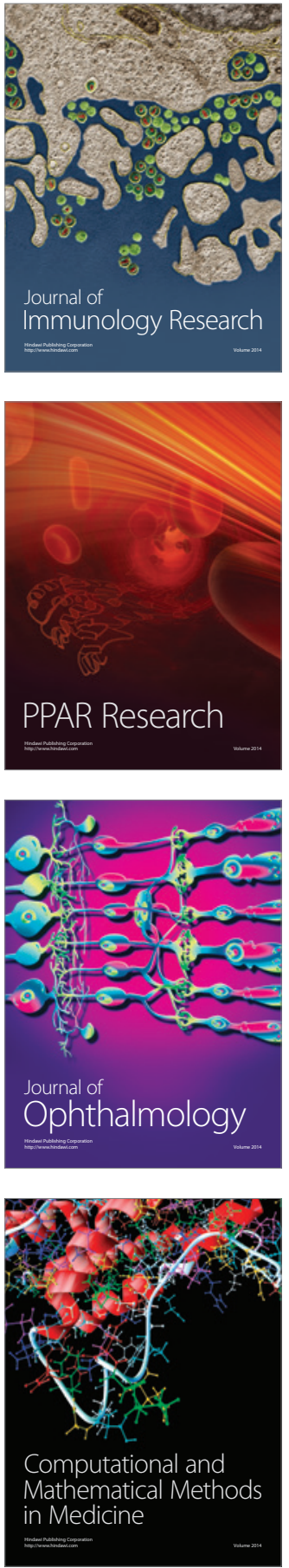

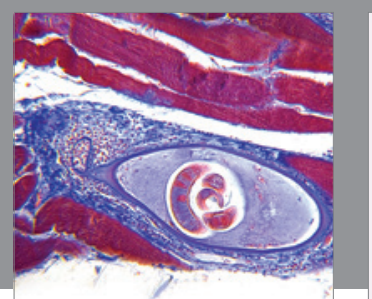

Gastroenterology Research and Practice

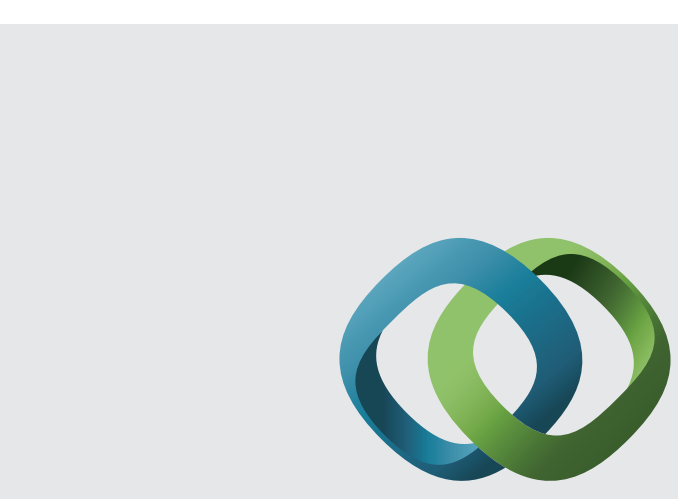

\section{Hindawi}

Submit your manuscripts at

http://www.hindawi.com
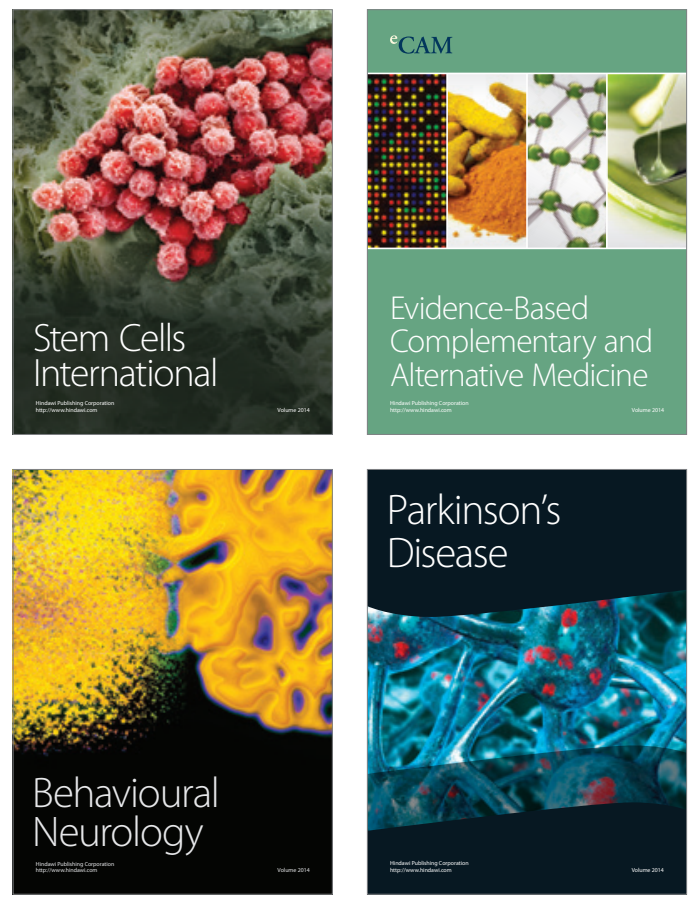
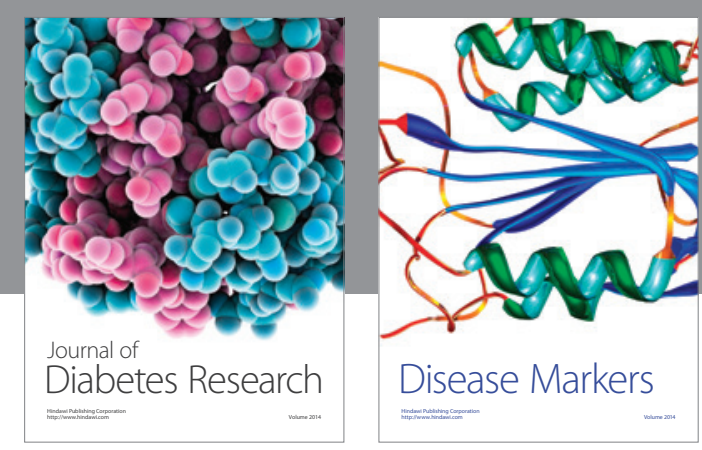

Disease Markers
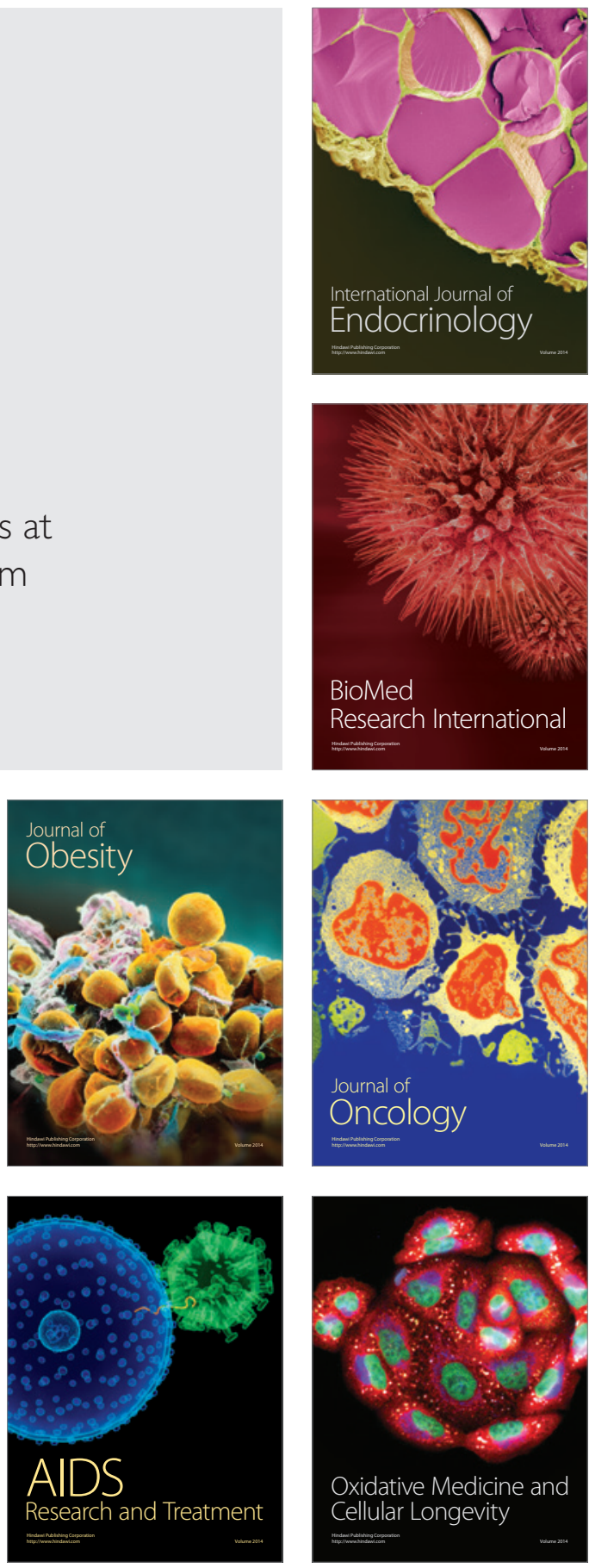